Pak. j. sci. ind. res. Ser. B: biol. sci. 2016 59(2) 59-68

\title{
Phosphorus Use Efficiency and Yield of Direct Seeded Rice and Wheat Influenced by Residues Incorporation and Phosphorus Application under Saline Soil
}

\author{
Imdad Ali Mahmood ${ }^{\mathrm{a} *}$, Arshad Ali ${ }^{\mathrm{a}}$, Armghan Shahzad ${ }^{\mathrm{b}}$ and Tariq Sultan ${ }^{\mathrm{a}}$ \\ ${ }^{a}$ Land Resources Research Institute, NARC, Islamabad-45500, Pakisktan \\ ${ }^{b}$ National Institute for Genomics and Advanced Biotechnology, NARC, Park Road, \\ Islamabad-45500, Pakistan
}

(received September 14, 2015; revised November 27, 2015; accepted December 23, 2015)

\begin{abstract}
A two years field study according to split plot design was conducted to investigate the impact of crop residue (CR) incorporation and $\mathrm{P}$ application $\left(0,40,80,120 \mathrm{~kg} \mathrm{P}_{2} \mathrm{O}_{5} / \mathrm{ha}\right)$ on $\mathrm{P}$ use efficiency and yield of direct seeded rice (DSR) and wheat grown under saline soil $\left(\mathrm{EC}_{\mathrm{e}}=4.59 \mathrm{dS} / \mathrm{m} ; \mathrm{pHs}=8.38\right.$; $\mathrm{SAR}=6.57\left(\mathrm{mmol}_{\mathrm{c}} / \mathrm{L}\right)^{1 / 2}$; extractable $\mathrm{P}=4.07 \mathrm{mg} / \mathrm{kg}$; texture $=$ sandy clay loam $)$, during the years 2011 and 2012. Planting of DSR (with and without crop residue incorporation @ 2 tonnes/ha) were placed in main plots and $\mathrm{P}$ application was in sub plots. Data on tillering, plant height, panicle length, 1000 grain weight, paddy and straw yields were collected. On an average of two years, maximum tillers (18), panicle length (33), grain/panicle (121) and paddy yield (3.26 t/ha) were produced with P application @ $80 \mathrm{~kg}$ $\mathrm{P}_{2} \mathrm{O}_{5}$ /ha along with CR incorporation. Similarly in case of wheat grown after DSR, maximum tillers (17), spike length (17), grains/panicle (66) and grain yield (3.56 t/ha) were produced with P application @ $80 \mathrm{~kg}$ $\mathrm{P}_{2} \mathrm{O}_{5} /$ ha along with $\mathrm{CR}$ incorporation. Although, the growth and yield contributing parameters with this treatment $\left(80 \mathrm{~kg} \mathrm{P}_{2} \mathrm{O}_{5} / \mathrm{ha}+\mathrm{CR}\right)$ performed statistically equal to $120 \mathrm{~kg} \mathrm{P}_{2} \mathrm{O}_{5} /$ ha without $\mathrm{CR}$ incorporation during both the years, but on an average of two years, grain yield of DSR and wheat was significantly superior (22 and 24\%, respectively) than that of higher P rate (120 kg/ha) without CR. Overall, continuous two years CR incorporation further increased (17\%) paddy yields during the follow up year of crop harvest. Higher $\mathrm{P}$ use efficiency and concentrations of $\mathrm{P}, \mathrm{K}^{+}$and $\mathrm{Ca}^{2+}$ in both DSR and wheat plant tissues was found where $80 \mathrm{~kg} \mathrm{P}_{2} \mathrm{O}_{5} /$ ha was applied along with $\mathrm{CR}$ incorporation or $120 \mathrm{~kg} \mathrm{P}_{2} \mathrm{O}_{5} /$ ha alone while $\mathrm{Na}^{+}$ and $\mathrm{Mg}^{2+}$ concentration decreased with $\mathrm{CR}$ incorporation and increasing P rate. An increasing trend in DSR paddy and wheat grain yields was observed with increasing the rate of $\mathrm{P}$ application without $\mathrm{CR}$ incorporation, however, it was not as much as that of $80 \mathrm{~kg} \mathrm{P}_{2} \mathrm{O}_{5} /$ ha application with CR incorporation and found to be superior than rest of the treatments during both study years.
\end{abstract}

Keywords: direct seeded rice, wheat, saline soil, crop residues, $\mathrm{P}$ application

\section{Introduction}

Soil salinity is a major problem in boosting up agricultural production throughout the world due to which million hectares of agricultural land are unable to produce potential crop yields. In regions such as Pakistan, over a quarter of the cultivatable land is occupied by medium to high salinity (Ahmad, 2002; Qureshi and Qadir, 1992). The problem is going faster because of heavy irrigations with brackish water which further decline the fertility of culturable lands and crop yields (Qadir, et al., 2003; Ahmad, 2002; Minhas, 1996; Ghafoor et al., 1992; Ahmad et al., 1990). Saline soils contain surplus soluble salts $\left(\mathrm{Cl}^{-}\right.$and $\mathrm{SO}_{4}^{--}$of $\mathrm{Na}^{+}, \mathrm{Ca}^{2+}$ and $\mathrm{Mg}^{2+}$ ) which cause high osmotic pressure and compound interactions of $\mathrm{Na}, \mathrm{Ca}$ and $\mathrm{K}$ (Maser et al., 2002). These

*Author for correspondence; E-mail: imdadlrri@gmail.com salts disturb the equilibrium in rhizosphere and affect crop productivity. Adequate plant nutrition may reduce ill effects of these ions thereby helping plants to improve their growth and productivity under such situations (Mahmood et al., 2013; Aslam et al., 2008; Ghafoor et al., 2004; Mahmood et al., 1994). Phosphorus availability in problem soils is affected by anion competition $\left(\mathrm{PO}_{4}^{-}\right.$and $\left.\mathrm{Cl}^{-}\right)$and many other interactions. In calcareous soils, applied $\mathrm{P}$ almost instantaneously gets fixed with lime due to the formation of insoluble dibasic calcium phosphate compounds. Much work has been reported regarding nutrient management for conventional crops to enhance their yields under unfavourable situation but much less exertion is reported on direct seeded rice under saline soils. Optimised P nutrition is critical for producing potential yields because it encourages healthy growth, such as development of strong root system, 
maximum tillering, upholds more flowering and seed formation (Sainio et al., 2006). Often P deficiency in rice is referred to as "hidden hunger" which causes poor tillering, slow leaf canopy expansion, poor grain formation and delayed maturity. In Pakistan, more than $90 \%$ soils are deficient in P (NFDC, 2012; 2010). Farming community in rice-wheat cropping system is facing water shortage, escalating fuel and high fertilizer cost (particularly of P fertilizers) and labour shortage during rice transplanting. About half of the rice cultivated area $(\sim 1.0 \mathrm{Mha})$ in Punjab province is salt-affected which has moderate to high salinity, high $\mathrm{pH}$ and shortage of good quality ground water causing $30-70 \%$ paddy yield reduction (Qayyum and Malik, 1988). Moreover, P availability in soluble orthophosphate form is a prevalent limitation under calcareous soils because it makes insoluble compounds and does not release plant available $\mathrm{P}$ even upon heavy irrigations. A large proportion of applied $\mathrm{P}$ in the soil becomes immobile due to this process (Mahmood et al., 2013; Rahmatullah et al., 1994; ) however, the plants readily utilize only $8-33 \%$ of applied $\mathrm{P}$ in the first growing season (Saleem et al., 1986). Hence, there is a need to increase the use of $\mathrm{P}$ fertilizers in order to guarantee food security for ever increasing populations.

Most of the farmers of rice-wheat cropping system burn wheat crop residues to prepare their lands for timely rice transplanting. This practice not only despoil the environment but also the precious source of plant nutrients (main source of organic material and the important constituent for soil health) are being smashed on large scale in this agricultural ecosystem. About 25\% of $\mathrm{N}$ and $\mathrm{P}, 50 \%$ of $\mathrm{S}$ and $75 \%$ of $\mathrm{K}$ uptake by cereal crops are retained in crop residues, making them valuable nutrient sources. Since large portion of nutrients taken up by plants remains in the straw, much of this can be recycled for subsequent crop growth after its decomposition (Mahmood et al., 2013; Byous et al., 2004). In many studies, impact of residues incorporation on soil health had been reported to increase the organic matter and nutrient status and hence crop yields improvement (Ali et al., 2012; Krishna et al., 2004; Eagle et al., 2000; Misra et al., 1996). Direct seeding of rice is a new and the most suitable technology for resource poor community of salt-affected lands for which transplanting labour cost, water and machinery tools expenses required during puddling and transplanting could be saved in addition to timely sowing of rice. Weedicide application can be done to control weeds at early stages of growth. The crop matures early as compared to traditional transplanting which further makes possible sowing of wheat crop well in time after the harvest of rice. Besides these benefits, there is much more plant population under direct seeded rice as compared to traditionally transplanted rice for which nutrients requirement could also be higher to produce potential yields.

A little work is reported regarding nutrient management particularly of $\mathrm{P}$ application for direct seeded rice in salt-affected soils of Pakistan. Therefore, keeping all these factors in mind, a long term field study was planned to investigate appropriate $\mathrm{P}$ dose for direct seeded rice along with wheat straw incorporating and their impact on paddy yield in a slightly saline soil of district Hafizabad, Pakistan.

\section{Materials and Methods}

A two year study using a permanent layout was conducted under marginal saline soil of rice-wheat cropping system at farmers field in Wachhoki Kalan, Kankah Dogran-Hafizabad Road, district Hafizabad, Pakistan $\left(\mathrm{EC}_{\mathrm{e}}=4.59 \mathrm{dS} / \mathrm{m} ; \mathrm{pH}_{\mathrm{s}}=8.38 ; \mathrm{SAR}=6.57\right.$ $\left(\mathrm{mmol}_{\mathrm{c}} / \mathrm{L}\right)^{1 / 2} ;$ extractable $\mathrm{P}=4.07 \mathrm{mg} / \mathrm{kg}$; texture $=$ sandy clay loam) during 2011 and 2012. The experiment was laid out according to split plot design with three replications. Planting methods; direct seeding with and without crop residue (wheat) incorporation (a) $2 \mathrm{t} / \mathrm{ha}$ were kept in main plots and various $\mathrm{P}$ doses $\left(0,40,80\right.$ and $\left.120 \mathrm{~kg} \mathrm{P}_{2} \mathrm{O}_{5} / \mathrm{ha}\right)$ were applied in sub plots.

Recommended basal dose of N@100 kg/ha (half at sowing time and remaining half at tillering stage) and $\mathrm{K} @ 50 \mathrm{~kg} / \mathrm{ha}$ as SOP were applied to all the plots at the time of sowing. Soaked seed (for $24 \mathrm{~h}$ ) of rice cv. Basmati-2000@40 kg/ha was broad-casted uniformly. The same inputs were applied to intermediate wheat crop. Effective weedicides were used to control weeds and the crop was grown to maturity. All agronomic requirements and plant protection measures were met throughout the growth period whenever required. Presowing soil samples (0-15 cm depth) were collected for the analysis of general soil characteristics (Table 1) according to the methods suggested by Ryan et al. (2001). Plant samples were collected at maturity for the determination of ionic concentration in tissues. 
Table 1. General characteristics of saline soil before initiation and after the study during 2011 and 2012 (Average of three repeats)

\begin{tabular}{|c|c|c|c|c|c|c|c|c|c|c|c|c|c|c|c|c|c|c|}
\hline \multirow{2}{*}{$\begin{array}{l}\mathrm{P}_{2} \mathrm{O}_{5} \\
\text { (kg/ha) }\end{array}$} & \multicolumn{3}{|c|}{$\mathrm{EC}_{\mathrm{e}}$} & \multicolumn{3}{|c|}{ SAR } & \multicolumn{3}{|c|}{$\mathrm{pH}$} & \multicolumn{3}{|c|}{$\mathrm{OM}$} & \multicolumn{3}{|c|}{$P$} & \multicolumn{3}{|c|}{$\mathrm{K}$} \\
\hline & $\overline{+C R}$ & $-\mathrm{CR}$ & Mean & $+\mathrm{CR}$ & $-\mathrm{CR}$ & Mean & $+\mathrm{CR}$ & $-\mathrm{CR}$ & Mean & $+\mathrm{CR}$ & $-\mathrm{CR}$ & Mean & $\overline{+C R}$ & $-\mathrm{CR}$ & Mean & $\overline{+\mathrm{CR}}$ & $-\mathrm{CR}$ & Mean \\
\hline \multicolumn{19}{|c|}{ Before sowing } \\
\hline 0 & $4.59^{\mathrm{NS}}$ & $4.58^{\mathrm{NS}}$ & $4.58^{\mathrm{NS}}$ & $6.57^{\mathrm{NS}}$ & 6.56 & $6.57^{\mathrm{NS}}$ & $8.38^{\mathrm{NS}}$ & $8.38^{\mathrm{NS}}$ & $8.38^{\mathrm{NS}}$ & $0.52^{\mathrm{NS}}$ & $0.54^{\mathrm{NS}}$ & $0.54^{\mathrm{NS}}$ & $4.08^{\mathrm{NS}}$ & $4.07^{\mathrm{NS}}$ & $4.07^{\mathrm{NS}}$ & $78.03^{\mathrm{NS}}$ & S $77.05^{\mathrm{NS}}$ & $\mathrm{S} 77.02^{\mathrm{NS}}$ \\
\hline 40 & 4.57 & 4.57 & 4.58 & 6.56 & 6.57 & 6.57 & 8.39 & 8.37 & 8.38 & 0.54 & 0.52 & 0.54 & 4.06 & 4.08 & 4.07 & 77.10 & 76.49 & 77.02 \\
\hline 80 & 4.58 & 4.59 & 4.58 & 6.57 & 6.56 & 6.57 & 8.38 & 8.38 & 8.38 & 0.53 & 0.54 & 0.54 & 4.07 & 4.07 & 4.07 & 79.09 & 77.02 & 77.02 \\
\hline 120 & 4.58 & 4.58 & 4.58 & 6.56 & 6.57 & 6.57 & 8.38 & 8.39 & 8.38 & 0.54 & 0.53 & 0.54 & 4.07 & 4.07 & 4.07 & 76.62 & 77.00 & 77.02 \\
\hline Mean & $4.58^{\mathrm{NS}}$ & 4.58 & - & $6.57^{\mathrm{NS}}$ & 6.57 & - & $8.38^{\mathrm{NS}}$ & 8.38 & - & $0.54^{\mathrm{NS}}$ & 0.54 & - & $4.07^{\mathrm{NS}}$ & 4.07 & - & $77.02^{\mathrm{NS}}$ & s 77.02 & - \\
\hline \multicolumn{19}{|c|}{ After harvest of final crop } \\
\hline 0 & $3.87 \mathrm{~d}$ & $4.58 \mathrm{a}$ & $4.22 \mathrm{~A}$ & $4.92 \mathrm{~d}$ & $6.57 \mathrm{a}$ & $5.74 \mathrm{~A}$ & $8.18 \mathrm{c}$ & $8.38 \mathrm{a}$ & $8.28 \mathrm{~A}$ & $0.68^{\mathrm{NS}}$ & $0.49^{\mathrm{NS}}$ & $0.59^{\mathrm{NS}}$ & Is $2.99 \mathrm{ef}$ & $1.28 \mathrm{f}$ & $2.13 \mathrm{D}$ & $95.26 \mathrm{a}$ & $78.86 \mathrm{~d}$ & $87.06 \mathrm{~A}$ \\
\hline 40 & $3.84 \mathrm{~d}$ & $4.51 \mathrm{~b}$ & 4.17B & $4.87 \mathrm{de}$ & $6.45 b$ & $5.66 \mathrm{~B}$ & $8.16 \mathrm{c}$ & $8.37 \mathrm{a}$ & 8.27B & 0.69 & 0.52 & 0.61 & $8.26 \mathrm{~cd}$ & $3.89 \mathrm{e}$ & $6.08 \mathrm{C}$ & $93.46 \mathrm{~b}$ & $75.73 \mathrm{e}$ & $84.60 \mathrm{~B}$ \\
\hline 80 & $3.69 \mathrm{f}$ & $4.42 \mathrm{c}$ & $4.06 \mathrm{C}$ & $4.82 \mathrm{e}$ & $6.42 \mathrm{bc}$ & $5.62 \mathrm{BC}$ & $8.11 \mathrm{e}$ & $8.34 \mathrm{~b}$ & $8.23 \mathrm{C}$ & 0.71 & 0.53 & 0.62 & $11.23 \mathrm{~b}$ & $6.86 \mathrm{~d}$ & $9.05 \mathrm{~B}$ & $88.48 \mathrm{c}$ & $73.86 \mathrm{e}$ & $81.17 \mathrm{C}$ \\
\hline 120 & $3.77 \mathrm{e}$ & $4.38 \mathrm{c}$ & $4.08 \mathrm{C}$ & $4.85 \mathrm{de}$ & $6.36 \mathrm{c}$ & $5.61 \mathrm{C}$ & $8.14 \mathrm{~d}$ & $8.32 b$ & $8.23 \mathrm{C}$ & 0.70 & 0.54 & 0.62 & $14.57 \mathrm{a}$ & $9.56 \mathrm{bc}$ & $12.06 \mathrm{~A}$ & $91.75 b$ & $70.82 \mathrm{f}$ & $81.29 \mathrm{C}$ \\
\hline Mean & $3.79 \mathrm{~B}$ & $4.47 \mathrm{~A}$ & - & $4.87 \mathrm{~B}$ & $6.45 \mathrm{~A}$ & - & $8.15 \mathrm{~B}$ & $8.35 \mathrm{~A}$ & - & $0.69 \mathrm{~A}$ & $0.52 \mathrm{~B}$ & - & $9.26 \mathrm{~A}$ & $5.40 \mathrm{~B}$ & - & $92.24 \mathrm{~A}$ & $74.82 \mathrm{~B}$ & - \\
\hline
\end{tabular}

$\mathrm{SAR}=$ sodium adsorption ratio; $\mathrm{OM}=$ organic matter (\%); Means followed by same letter (s) do not differ significantly at $\mathrm{P} \leq 0.05 \mathrm{NS}=$ non-significant.

Dried and ground samples were digested in perchloricnitric acid $(2: 11 \mathrm{~N})$ mixture (Rhoades, 1982) to estimate $\mathrm{P}, \mathrm{K}^{+}, \mathrm{Na}^{+}, \mathrm{Ca}^{2+}$ and $\mathrm{Mg}^{2+}$ concentrations by spectronic-20 and atomic absorption spectrophotometer. At maturity, the crops were harvested and agronomic data on fertile tillers, plant height, panicle length, grains/panicle, 1000-grain weight, paddy and straw yields were recorded. Phosphorus use efficiency (PUE) was computed by using the following formula as suggested by Fageria et al., (1997):

$$
\operatorname{PUE}(\%)=\frac{\left(\mathrm{TPU}_{\mathrm{F}}\right)-\left(\mathrm{TPU}_{\mathrm{C}}\right)}{\mathrm{P} \operatorname{dose}(\mathrm{kg} / \mathrm{ha})} \times 100
$$

where:

$\mathrm{TPU}_{\mathrm{F}}=$ total P uptake $(\mathrm{kg} / \mathrm{ha})$ in fertilized plots and $\mathrm{TPU}_{\mathrm{C}}=$ total $\mathrm{P}$ uptake $(\mathrm{kg} / \mathrm{ha})$ in control plots.

Total PU was calculated as:

$$
\operatorname{TPU}(\mathrm{kg} / \mathrm{ha})=\frac{\begin{array}{c}
\text { P contents }(\%) \text { in plant tissues } \\
\text { (dry matter }) \times \text { yield }(\mathrm{kg} / \mathrm{ha})
\end{array}}{100} \ldots \ldots . . .
$$

The Eq. 2 was used to calculate $\mathrm{TPU}_{\mathrm{F}}$ and $\mathrm{TPU}_{\mathrm{C}}$ separately for putting in Eq. 1.

The data thus, collected were subjected to statistical analysis using software package MSTAT-C and treatment means were compared using least significant difference (LSD) at 5\% probability level (Gomez and Gomez, 1984).

\section{Results and Discussion}

Growth and yield of DSR. On an average of two years DSR crop data, maximum fertile tillers (19), panicle length $(34 \mathrm{~cm})$, grain/panicle (121) and paddy yield (3.26 t/ha) were produced with $80 \mathrm{~kg} \mathrm{P}_{2} \mathrm{O}_{5} /$ ha application along with $\mathrm{CR}$ incorporation (Table 2). Paddy yield obtained from the plots fertilized with $80 \mathrm{~kg} \mathrm{P}_{2} \mathrm{O}_{5} / \mathrm{ha}$ under $\mathrm{CR}$ incorporation was statistically similar to paddy yield obtained under higher $\mathrm{P}$ application rate (120 kg P $\mathrm{P}_{2} /$ ha) with no CR incorporation, however, paddy yield obtained from the plots fertilized with rate of $80 \mathrm{~kg} \mathrm{P}_{2} \mathrm{O}_{5} /$ ha under $\mathrm{CR}$ incorporation was otherwise superior $(6 \%)$ than that of obtained under higher $\mathrm{P}$ rate (120 kg $\mathrm{P}_{2} \mathrm{O}_{5} /$ ha) without CR. Overall, paddy yield produced by this treatment $\left(80 \mathrm{~kg} \mathrm{P}_{2} \mathrm{O}_{5} / \mathrm{ha}\right)$ showed $22 \%$ additional yield over control $(0 \mathrm{~kg} \mathrm{P} / \mathrm{ha}+\mathrm{CR})$. During second year of DSR, the lower dose of $\mathrm{P}$ (40 $\mathrm{kg} \mathrm{P}_{2} \mathrm{O}_{5} / \mathrm{ha}+\mathrm{CR}$ ) performed statistically equal to higher $\mathrm{P}$ rate $\left(120 \mathrm{~kg} \mathrm{P}_{2} \mathrm{O}_{5} /\right.$ ha) without CR incorporation. On the average, continuous two year $\mathrm{CR}$ incorporation further increased $17 \%$ paddy yield during the follow up year of crop harvest as compared to previous crop harvest under CR incorporation. 
Growth and yield of wheat crop. Minimum number of productive tillers (9), spike length $(12 \mathrm{~cm})$, grain/spike (33) and grain yield (2.87 t/ha) were produced from the plots where no $\mathrm{P}$ was applied under $\mathrm{CR}$ incorporation however, these parameters were significantly higher than that of control treatment $\left(0 \mathrm{~kg} \mathrm{P}_{2} \mathrm{O}_{5} / \mathrm{ha}\right)$ without $\mathrm{CR}$ incorporation (Table 3). A considerable increase in growth and yield contributing parameters was observed with $\mathrm{P}$ fertilization and $\mathrm{CR}$ incorporation. Maximum fertile tillers (17), spike length $(17 \mathrm{~cm})$, grain/panicle (56) and grain yield (3.56 t/ha) was produced with $\mathrm{P}$ application (@80 kg $\mathrm{P}_{2} \mathrm{O}_{5} / \mathrm{ha}$ ) along with CR incorporation. Grain yield harvested with $80 \mathrm{~kg} \mathrm{P}_{2} \mathrm{O}_{5} / \mathrm{ha}$ application and $\mathrm{CR}$ incorporation was $7 \%$ higher than extended $\mathrm{P}$ application rate $\left(120 \mathrm{~kg} \mathrm{P}_{2} \mathrm{O}_{5} / \mathrm{ha}\right)$ without $\mathrm{CR}$ incorporation. Wheat grain yield harvested with $40 \mathrm{~kg} \mathrm{P}_{2} \mathrm{O}_{5} /$ ha application and $\mathrm{CR}$ incorporation was also statistically similar to higher $\mathrm{P}$ rate $\left(120 \mathrm{~kg} \mathrm{P}_{2} \mathrm{O}_{5} / \mathrm{ha}\right)$ without $\mathrm{CR}$ incorporation. When $\mathrm{P}$ was applied without
CR incorporation, maximum grain yield was obtained with higher rate of $\mathrm{P}\left(120 \mathrm{~kg} \mathrm{P}_{2} \mathrm{O}_{5} / \mathrm{ha}\right)$ but was not again as much as harvested @ $80 \mathrm{~kg} \mathrm{P}_{2} \mathrm{O}_{5} /$ ha under CR incorporation. From our data, it can be seen that the paddy yield was $2.9 \mathrm{t}$ /ha during the first year of experimentation while it was $3.5 \mathrm{t} /$ ha during second year (Table 2 ). In comparison to regular practice of transplanting where the national yield is $2.4 \mathrm{t} / \mathrm{ha}$ on normal soils (GOP, 2014), yield data of present study is promising with the yield increase of $21 \%$ for first year and $45 \%$ for second year.

Ionic concentration and PUE of DSR and wheat. It is evident from the data in Table 4-5 that high $\mathrm{Na}^{+}$ and $\mathrm{Mg}^{2+}$ while low $\mathrm{K}^{+}$and $\mathrm{Ca}^{2+}$ concentrations were determined from both DSR and wheat plant tissues where no $\mathrm{P}$ was applied without $\mathrm{CR}$ incorporation. Phosphorus application and $\mathrm{CR}$ incorporation significantly reduced saline ions $\left(\mathrm{Na}^{+}\right.$and $\left.\mathrm{Mg}^{2+}\right)$

Table 2. Growth and yield of direct seeded rice (DSR) influenced by P application and crop residues (CR) incorporation under saline soil (Average of three repeats)

\begin{tabular}{|c|c|c|c|c|c|c|c|c|c|c|c|c|}
\hline \multirow{2}{*}{$\begin{array}{l}\mathrm{P}_{2} \mathrm{O}_{5} \\
(\mathrm{~kg} / \mathrm{ha})\end{array}$} & \multicolumn{2}{|c|}{ Tillers } & \multicolumn{2}{|c|}{ Plant height $(\mathrm{cm})$} & \multicolumn{2}{|c|}{ Panicle length $(\mathrm{cm})$} & \multicolumn{2}{|c|}{ Grain/panicle } & \multicolumn{2}{|c|}{ Paddy yield (t/ha) } & \multicolumn{2}{|c|}{ Straw yield $(\mathrm{t} / \mathrm{ha})$} \\
\hline & $\overline{+\mathrm{CR}}$ & $-\mathrm{CR}$ & $+\mathrm{CR}$ & $-\mathrm{CR}$ & $\overline{+\mathrm{CR}}$ & $-\mathrm{CR}$ & $+\mathrm{CR}$ & $-\mathrm{CR}$ & $\overline{+\mathrm{CR}}$ & $-\mathrm{CR}$ & $+\mathrm{CR}$ & $-\mathrm{CR}$ \\
\hline \multicolumn{13}{|l|}{2011} \\
\hline 0 & $13.67 \mathrm{bc}$ & $8.00 \mathrm{~d}$ & $126.33 \mathrm{a}$ & $118.00 \mathrm{~b}$ & $26.00 \mathrm{bc}$ & $17.67 \mathrm{~d}$ & $108.67 b c$ & $78.33 \mathrm{~d}$ & $2.497 \mathrm{~cd}$ & $1.887 \mathrm{e}$ & $6.430 \mathrm{ab}$ & $4.760 \mathrm{c}$ \\
\hline 40 & $14.00 \mathrm{bc}$ & $12.33 \mathrm{c}$ & $129.00 \mathrm{a}$ & $119.67 \mathrm{~b}$ & $27.00 \mathrm{bc}$ & $22.00 \mathrm{c}$ & $110.67 b$ & $99.33 \mathrm{c}$ & $2.637 \mathrm{bc}$ & $2.215 \mathrm{~d}$ & $6.590 \mathrm{a}$ & $5.657 b$ \\
\hline 80 & $17.67 \mathrm{a}$ & $15.67 \mathrm{ab}$ & $130.00 \mathrm{a}$ & $120.67 \mathrm{~b}$ & $30.67 \mathrm{a}$ & $24.67 \mathrm{bc}$ & $119.33 \mathrm{a}$ & $105.67 \mathrm{bc}$ & $2.987 \mathrm{a}$ & $2.707 a b c$ & $6.980 \mathrm{a}$ & $6.523 \mathrm{ab}$ \\
\hline 120 & $16.00 \mathrm{ab}$ & $16.00 \mathrm{ab}$ & $130.00 \mathrm{a}$ & $120.67 b$ & $28.00 \mathrm{ab}$ & $27.00 \mathrm{ab}$ & $115.67 \mathrm{a}$ & $114.33 \mathrm{ab}$ & $2.852 \mathrm{ab}$ & $2.817 \mathrm{abc}$ & $6.830 \mathrm{a}$ & $6.737 \mathrm{a}$ \\
\hline LSD & \multicolumn{2}{|c|}{2.8362} & \multicolumn{2}{|c|}{4.6787} & \multicolumn{2}{|c|}{3.1727} & \multicolumn{2}{|c|}{8.3600} & \multicolumn{2}{|c|}{0.2639} & \multicolumn{2}{|c|}{0.9532} \\
\hline \multicolumn{13}{|l|}{2012} \\
\hline 0 & $14.67 \mathrm{bc}$ & $9.33 \mathrm{~d}$ & $125.67 \mathrm{~b}$ & $98.00 \mathrm{~d}$ & $29.33 b c$ & $17.00 \mathrm{e}$ & $105.67 \mathrm{de}$ & $86.00 \mathrm{f}$ & $2.873 \mathrm{bc}$ & $1.560 \mathrm{e}$ & $7.223 b$ & $5.090 \mathrm{~d}$ \\
\hline 40 & $15.00 \mathrm{bc}$ & $13.00 \mathrm{c}$ & $127.00 \mathrm{ab}$ & $114.00 \mathrm{c}$ & $31.00 \mathrm{~b}$ & $24.00 \mathrm{~d}$ & $113.33 \mathrm{bc}$ & $99.00 \mathrm{e}$ & $3.103 \mathrm{abc}$ & $2.127 \mathrm{~d}$ & $7.313 b$ & $5.750 \mathrm{c}$ \\
\hline 80 & $18.67 \mathrm{a}$ & $15.67 \mathrm{ab}$ & $133.67 \mathrm{a}$ & $115.33 \mathrm{c}$ & $35.67 \mathrm{a}$ & $25.33 \mathrm{~cd}$ & $122.67 \mathrm{a}$ & $109.00 \mathrm{~cd}$ & $3.537 \mathrm{a}$ & $2.723 c$ & $7.913 \mathrm{a}$ & $6.542 b$ \\
\hline 120 & $16.33 \mathrm{ab}$ & $16.33 \mathrm{ab}$ & $129.00 \mathrm{ab}$ & $116.33 \mathrm{c}$ & $31.67 \mathrm{~b}$ & $26.67 \mathrm{bcd}$ & $118.67 \mathrm{ab}$ & $115.00 \mathrm{ab}$ & $3.308 \mathrm{ab}$ & $3.307 \mathrm{ab}$ & $7.510 \mathrm{ab}$ & $6.730 \mathrm{~b}$ \\
\hline LSD & \multicolumn{2}{|c|}{2.4983} & \multicolumn{2}{|c|}{7.7940} & \multicolumn{2}{|c|}{3.7621} & \multicolumn{2}{|c|}{5.6257} & \multicolumn{2}{|c|}{0.5246} & \multicolumn{2}{|c|}{0.5817} \\
\hline
\end{tabular}

Means followed by same letter (s) do not differ significantly at $\mathrm{P} \leq 0.05$.

Table 3. Growth and yield of wheat influenced by P application and CR incorporation under saline soil (average of three repeats)

\begin{tabular}{|c|c|c|c|c|c|c|c|c|c|c|c|c|}
\hline \multirow{2}{*}{$\begin{array}{l}\mathrm{P}_{2} \mathrm{O}_{5} \\
(\mathrm{~kg} / \mathrm{ha})\end{array}$} & \multicolumn{2}{|c|}{ Tillers } & \multicolumn{2}{|c|}{ Plant height $(\mathrm{cm})$} & \multicolumn{2}{|c|}{ Spike/length $(\mathrm{cm})$} & \multicolumn{2}{|c|}{ Grain/spike } & \multicolumn{2}{|c|}{ Grain yield (t/ha) } & \multicolumn{2}{|c|}{ Straw yield $(\mathrm{t} / \mathrm{ha})$} \\
\hline & $+\mathrm{CR}$ & $-\mathrm{CR}$ & $+\mathrm{CR}$ & $-\mathrm{CR}$ & $+\mathrm{CR}$ & $-\mathrm{CR}$ & $+\mathrm{CR}$ & $-\mathrm{CR}$ & $+\mathrm{CR}$ & $-\mathrm{CR}$ & $+\mathrm{CR}$ & $-\mathrm{CR}$ \\
\hline 0 & $9.33 d$ & $6.67 \mathrm{e}$ & $81.00 \mathrm{ab}$ & $71.00 \mathrm{~b}$ & $12.67 \mathrm{~cd}$ & $9.67 \mathrm{e}$ & $33.00 \mathrm{c}$ & $20.67 \mathrm{~d}$ & $2.652 \mathrm{c}$ & $1.591 \mathrm{e}$ & $5.936 \mathrm{ab}$ & $3.476 \mathrm{~d}$ \\
\hline 40 & $12.33 \mathrm{c}$ & $8.67 \mathrm{de}$ & $88.33 a$ & $74.33 b$ & $14.33 \mathrm{bc}$ & $11.00 \mathrm{de}$ & $46.67 b$ & $42.67 b$ & $3.194 \mathrm{abc}$ & $2.054 \mathrm{~d}$ & $6.314 \mathrm{ab}$ & $4.433 \mathrm{c}$ \\
\hline 80 & $17.00 \mathrm{a}$ & $14.00 \mathrm{bc}$ & $89.67 \mathrm{a}$ & $78.67 \mathrm{ab}$ & $17.33 \mathrm{a}$ & $12.67 \mathrm{~cd}$ & $56.33 \mathrm{a}$ & $43.67 b$ & $3.560 \mathrm{a}$ & $2.877 \mathrm{bc}$ & $6.676 \mathrm{a}$ & $5.447 \mathrm{~b}$ \\
\hline 120 & $14.33 \mathrm{bc}$ & $16.33 \mathrm{ab}$ & $89.33 \mathrm{a}$ & $79.00 \mathrm{ab}$ & $15.33 \mathrm{ab}$ & $16.33 \mathrm{ab}$ & $48.33 b$ & $46.00 \mathrm{~b}$ & $3.259 \mathrm{ab}$ & $3.320 \mathrm{ab}$ & $6.377 \mathrm{ab}$ & $6.547 \mathrm{a}$ \\
\hline LSD & \multicolumn{2}{|c|}{2.4807} & \multicolumn{2}{|c|}{9.2534} & \multicolumn{2}{|c|}{2.5848} & \multicolumn{2}{|c|}{6.2967} & \multicolumn{2}{|c|}{0.4200} & \multicolumn{2}{|c|}{0.9954} \\
\hline
\end{tabular}

Means followed by same letter (s) do not differ significantly at $\mathrm{P} \leq 0.05$. 
concentration and improved $\mathrm{K}^{+}$and $\mathrm{Ca}^{2+}$ in both DSR and wheat crop plants. The data indicated that $\mathrm{P}$ application particularly with CR incorporation considerably decreased $\mathrm{Na}^{+}$concentration and increased $\mathrm{K}^{+}$and $\mathrm{Ca}^{2+}$ concentration in plant tissues. Phosphorus application still at lower rate $\left(40 \mathrm{~kg} \mathrm{P}_{2} \mathrm{O}_{5} /\right.$ ha) along with $\mathrm{CR}$ incorporation performed better or even statistically equal to higher dose of $\mathrm{P}\left(120 \mathrm{~kg} \mathrm{P}_{2} \mathrm{O}_{5} /\right.$ ha $)$ without CR incorporation.

Maximum PUE in case of DSR (26\%) and wheat (23\%) was reflected from the plots treated with $80 \mathrm{~kg} \mathrm{P}_{2} \mathrm{O}_{5} / \mathrm{ha}$ application under CR incorporation (Fig. 1) which was comparable with higher $\mathrm{P}$ dose $\left(120 \mathrm{~kg} \mathrm{P}_{2} \mathrm{O}_{5} / \mathrm{ha}\right)$ under no $\mathrm{CR}$ incorporation. Under $\mathrm{CR}$ incorporation, further increase in $\mathrm{P}$ application $\left(120 \mathrm{~kg} \mathrm{P}_{2} \mathrm{O}_{5} / \mathrm{ha}\right.$ ) did not show significant difference in PUE by both the crops. On the

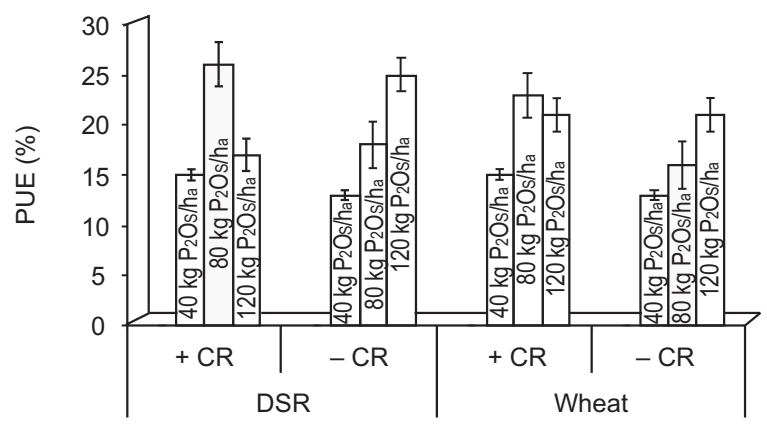

Fig. 1. PUE (\%) of direct seeded rice and wheat as influenced by crop residues incorporation and $\mathrm{P}$ application under saline soil.

other hand, when $\mathrm{P}$ was applied without $\mathrm{CR}$ incorporation, maximum PUE was determined from the treatments

Table 4. Ionic concentration (\%) in DSR plant tissues affected by $\mathrm{P}$ application and CR incorporation under saline soil (Average of three repeats)

\begin{tabular}{|c|c|c|c|c|c|c|c|c|}
\hline \multirow[t]{2}{*}{$\mathrm{P}_{2} \mathrm{O}_{5}(\mathrm{~kg} / \mathrm{ha})$} & \multicolumn{2}{|c|}{$\mathrm{K}^{+}$} & \multicolumn{2}{|c|}{$\mathrm{Na}^{+}$} & \multicolumn{2}{|c|}{$\mathrm{Ca}^{2+}$} & \multicolumn{2}{|c|}{$\mathrm{Mg}^{2+}$} \\
\hline & $+\mathrm{CR}$ & $-\mathrm{CR}$ & $+\mathrm{CR}$ & $-\mathrm{CR}$ & $\overline{+\mathrm{CR}}$ & $-\mathrm{CR}$ & $+\mathrm{CR}$ & $-\mathrm{CR}$ \\
\hline \multicolumn{9}{|c|}{2011} \\
\hline 0 & $1.906 \mathrm{e}$ & $1.827 \mathrm{e}$ & $0.276 b c$ & $0.459 \mathrm{a}$ & $0.196 \mathrm{c}$ & $0.091 \mathrm{~d}$ & $0.196 \mathrm{~b}$ & $0.389 \mathrm{a}$ \\
\hline 40 & $2.268 \mathrm{de}$ & $2.167 \mathrm{de}$ & $0.266 b c$ & $0.315 b$ & $0.234 \mathrm{c}$ & $0.212 \mathrm{c}$ & $0.195 b$ & $0.374 \mathrm{a}$ \\
\hline 80 & $3.445 \mathrm{a}$ & $2.555 \mathrm{~cd}$ & $0.144 \mathrm{e}$ & $0.230 \mathrm{~cd}$ & $0.322 \mathrm{a}$ & $0.247 \mathrm{bc}$ & $0.141 \mathrm{~b}$ & $0.390 \mathrm{a}$ \\
\hline 120 & $2.920 \mathrm{~b}$ & $2.992 b$ & $0.178 \mathrm{de}$ & $0.209 \mathrm{cde}$ & $0.298 \mathrm{ab}$ & $0.320 \mathrm{a}$ & $0.135 b$ & $0.355 \mathrm{a}$ \\
\hline \multirow[t]{2}{*}{ LSD } & \multicolumn{2}{|c|}{0.4237} & \multicolumn{2}{|c|}{0.0473} & \multicolumn{2}{|c|}{0.0618} & \multicolumn{2}{|c|}{0.0738} \\
\hline & \multicolumn{8}{|c|}{2012} \\
\hline 0 & 2.016de & $1.666 \mathrm{e}$ & $0.225 b c$ & $0.419 \mathrm{a}$ & $0.126 \mathrm{~d}$ & $0.085 \mathrm{e}$ & $0.120 \mathrm{c}$ & $0.215 \mathrm{a}$ \\
\hline 40 & $2.748 \mathrm{bc}$ & $2.044 \mathrm{de}$ & $0.178 \mathrm{~cd}$ & $0.290 \mathrm{ab}$ & $0.197 \mathrm{c}$ & $0.116 \mathrm{de}$ & $0.115 \mathrm{~cd}$ & $0.174 \mathrm{~b}$ \\
\hline 80 & $3.645 \mathrm{a}$ & $2.289 \mathrm{~cd}$ & $0.138 \mathrm{~d}$ & $0.228 b c$ & $0.291 \mathrm{a}$ & $0.196 \mathrm{c}$ & $0.094 \mathrm{~d}$ & $0.143 c$ \\
\hline 120 & $3.053 b$ & $2.739 \mathrm{bc}$ & $0.184 \mathrm{~cd}$ & $0.199 \mathrm{~cd}$ & $0.249 b$ & $0.276 \mathrm{ab}$ & $0.107 \mathrm{~d}$ & $0.106 \mathrm{~d}$ \\
\hline LSD & \multicolumn{2}{|c|}{0.4977} & \multicolumn{2}{|c|}{0.0734} & \multicolumn{2}{|c|}{0.0331} & \multicolumn{2}{|c|}{0.0275} \\
\hline
\end{tabular}

Means followed by same letter (s) do not differ significantly at $\mathrm{P} \leq 0.05$.

Table 5. Ionic concentration (\%) in wheat plant tissues influenced by $\mathrm{P}$ application and crop residues (CR) incorporation under saline soil (Average of three repeats)

\begin{tabular}{|c|c|c|c|c|c|c|c|c|}
\hline \multirow[t]{2}{*}{$\mathrm{P}_{2} \mathrm{O}_{5}(\mathrm{~kg} / \mathrm{ha})$} & \multicolumn{2}{|c|}{$\mathrm{K}^{+}$} & \multicolumn{2}{|c|}{$\mathrm{Na}^{+}$} & \multicolumn{2}{|c|}{$\mathrm{Ca}^{2+}$} & \multicolumn{2}{|c|}{$\mathrm{Mg}^{2+}$} \\
\hline & $+\mathrm{CR}$ & $-\mathrm{CR}$ & $+\mathrm{CR}$ & $-\mathrm{CR}$ & $+\mathrm{CR}$ & $-\mathrm{CR}$ & $+\mathrm{CR}$ & $-\mathrm{CR}$ \\
\hline \multicolumn{9}{|l|}{ Straw } \\
\hline 0 & $1.249 \mathrm{c}$ & $1.163 \mathrm{c}$ & $0.136 \mathrm{bc}$ & $0.221 \mathrm{a}$ & $0.124 \mathrm{~d}$ & $0.105 \mathrm{~d}$ & $0.143 b c$ & $0.216 \mathrm{a}$ \\
\hline 40 & $1.896 \mathrm{ab}$ & $1.148 \mathrm{c}$ & $0.104 \mathrm{de}$ & $0.207 \mathrm{a}$ & $0.211 b$ & $0.177 \mathrm{c}$ & $0.135 \mathrm{bcd}$ & $0.198 \mathrm{a}$ \\
\hline 80 & $2.100 \mathrm{a}$ & $1.800 \mathrm{~b}$ & $0.089 \mathrm{e}$ & $0.169 b$ & $0.293 \mathrm{a}$ & $0.205 b c$ & $0.106 \mathrm{~cd}$ & $0.149 \mathrm{~b}$ \\
\hline 120 & $1.923 \mathrm{ab}$ & $1.874 \mathrm{ab}$ & $0.097 \mathrm{e}$ & $0.135 \mathrm{~cd}$ & $0.219 b$ & $0.222 b$ & $0.121 \mathrm{bcd}$ & $0.102 d$ \\
\hline LSD & \multicolumn{2}{|c|}{0.2321} & \multicolumn{2}{|c|}{0.0310} & \multicolumn{2}{|c|}{0.0329} & \multicolumn{2}{|c|}{0.0403} \\
\hline
\end{tabular}

Means followed by same letter(s) do not differ significantly at $\mathrm{P} \leq 0.05$. 
where $\mathrm{P}$ was applied at higher rate $\left(120 \mathrm{~kg} \mathrm{P}_{2} \mathrm{O}_{5} / \mathrm{ha}\right)$ and was not as much of $\mathrm{P}$ application @ $80 \mathrm{~kg} \mathrm{P}_{2} \mathrm{O}_{5} / \mathrm{ha}$ under CR incorporation.

Effect of CR on fertility status and saline soil amelioration. The data in Table 1 shows that at the end of study, there was a significant improvement in organic matter content as well as $\mathrm{P}$ and $\mathrm{K}$ availability owing to continuous $\mathrm{CR}$ incorporation and $\mathrm{P}$ fertilization. Maximum SOM and P, K contents were recorded with increased $\mathrm{P}$ application rate under $\mathrm{CR}$ incorporation in saline soil.

After the harvest of final DSR crop, overall 21\% decline in ECe with CR incorporation was observed as compared to before the sowing of DSR with CR incorporation (Table 1). Increasing the rate of $P$ application under $C R$ incorporation also caused a significant reduction in ECe being the maximum decline with higher rates of $\mathrm{P}_{2} \mathrm{O}_{5}(80$ and $120 \mathrm{~kg} / \mathrm{ha}$ ) after completion of study. Overall, ECe fell down to $3.79 \mathrm{dS} / \mathrm{m}$ from $4.58 \mathrm{dS} / \mathrm{m}$ due to continuous $\mathrm{CR}$ incorporation and was fairly below the permissible limit. Similarly, under CR incorporation, there was also a considerable reduction in the value of SAR from 6.57 before crop sowing to $4.87\left(\mathrm{mmol}_{\mathcal{C}} / \mathrm{L}\right)^{1 / 2}$ after the harvest of third season DSR crop. A minimum mean value of $\mathrm{pH}(8.15)$ was observed from the plots incorporated with CR. Decrease in $\mathrm{pH}$ value under $\mathrm{CR}$ incorporation along with $\mathrm{P}$ application was slightly higher as compared to control $\left(0 \mathrm{~kg} \mathrm{P}_{2} \mathrm{O}_{5} / \mathrm{ha}\right)$ plots.

Growth and yield of DSR and wheat. Improved growth and yield of DSR and wheat with adequate $P$ application and $\mathrm{CR}$ incorporation was possibly as a result of $\mathrm{CR}$ incorporation that positively contributed in growth and yield of DSR particularly during second year. This was apparently due to complete decomposition and mineralization of added $\mathrm{CR}$ which provided healthy environment owing to perfect soil physical condition by ameliorating the adverse effects of hazardous ions (Table 1). Moreover, water and $\mathrm{P}$ retention capacity might have also been improved due to added CR that retained comparatively excess moisture and $\mathrm{P}$ availability for a longer time. Besides, fabrication of acid farming substances by microbial activities and partial pressure of $\mathrm{CO}_{2}$ released during $\mathrm{CR}$ decomposition decreased soil $\mathrm{pH}$ and enhanced $\mathrm{P}$ availability and other necessary plant nutrients which encouraged healthy plant growth and hence yields. Similar observations have also been documented by
Mahmood et al. (2013), Danga and Wakindiki, (2009), Mishra et al. (2006) and Rath et al. (2005). Further, adequate $\mathrm{P}$ fertilization promoted vigorous early plant growth, improved tillering and increased number of grains per panicle which eventually produced better yield of DSR (Arshadullah et al., 2012; Sainio et al., 2006). The increase in yield due to $C R$ incorporation as well as $\mathrm{P}$ application have also been well documented by Ali et al. (2012), Aslam et al. (2008), Krishna et al. (2004), Sharma and Prasad, (2003), Slaton et al. (2002), Eagle et al. (2000).

A significant increase in growth and yield contributing parameters of wheat grown after DSR was observed with $\mathrm{P}$ fertilization and $\mathrm{CR}$ incorporation. This was definitely attributable to $\mathrm{CR}$ incorporation that improved fertility status (Table 1) and consequently enhanced PUE (Fig. 1). Besides, adequate $\mathrm{P}$ application and its judicious utilization further facilitated to improve growth and yield. As it has been discussed earlier, that CR incorporation upon decomposition substantially changed the nutrient balance in rhizosphere which facilitated the plant roots to access the nutrients especially phosphorus. Growing plant roots and some microorganisms involved in decomposition of plant residues also discharge acid farming substances in the vicinity which could increase P solubility and its availability (Mahmood et al., 2013; Rehim et al., 2010).

Conversely, minimum number of productive tillers, panicle and spike lengths, DSR grains/panicle, wheat grains/spike and yields in case of control $\left(0 \mathrm{~kg} \mathrm{P}_{2} \mathrm{O}_{5} / \mathrm{ha}\right)$ of both DSR and wheat crops were due to P deficiency that directly distorted the normal tillering and grain formation by inhibiting their materialization and hence reduced yields (Rehim et al., 2010; Delgado et al., 2002). When $P$ was applied without $C R$ incorporation, higher grain yield was obtained with increasing $P$ rate and was maximum where P was applied @ $120 \mathrm{~kg}$ $\mathrm{P}_{2} \mathrm{O}_{5} /$ ha. Better crop growth and yield with maximum $\mathrm{P}$ application (120 kg $\mathrm{P}_{2} \mathrm{O}_{5} / \mathrm{ha}$ ) under no CR incorporation could be due to the reason that crops grown under salt-affected soils demands relatively higher nutrition to reach the potential yields (Aslam et al., 2008; Mahmood et al., 1994). However, this yield harvested with $120 \mathrm{~kg} \mathrm{P}_{2} \mathrm{O}_{5} /$ ha under no CR incorporation was $6 \%$ less than that obtained from $80 \mathrm{~kg} \mathrm{P}_{2} \mathrm{O}_{5} /$ ha with $\mathrm{CR}$ incorporation. It is obvious from the data in Table 2 that during second year of DSR grown under CR 
incorporation, even lower $\mathrm{P}$ rate $\left(40 \mathrm{~kg} \mathrm{P}_{2} \mathrm{O}_{5} / \mathrm{ha}\right)$ performed statistically equal to higher $P$ rate $(120 \mathrm{~kg}$ $\mathrm{P}_{2} \mathrm{O}_{5} / \mathrm{ha}$ ). This was most probably due to enhanced $\mathrm{P}$ availability in acidified rhizosphere as a result of microbial activities during CR decomposition. Moreover, fully decomposed $\mathrm{CR}$ and mineralization during subsequent wheat and DSR growing season presumably enriched the soil with mineral nutrients that contributed to a large extent in producing the maximum grain yields. Further, the longer root system in wheat crop might have absorbed nutrients from deeper soil layers to ensure healthy crop growth and hence yield. There are several reports that signify the role of $\mathrm{P}$ application in the enhancement of crop yields (Rehim et al., 2010; Danga and Wakindiki, 2009; Van der Eijk et al., 2006). Advantages of crop residue incorporation in soil and integrated nutrient management have been widely discussed by Ali et al. (2012); Shiva et al. (2012), Byous et al. (2004). Similar conclusions have also been reported by Yadvinder et al. (2004), Kharub et al. (2004), Sharma and Prasad (2003), Abid et al. (2002). Consequently, a substantial amount of $\mathrm{P}$ requirement for crop growth could be met by the incorporation of CR. Adequate $\mathrm{P}$ application approach on problem soils within the rice-wheat cropping system have to guarantee high and sustainable food grain production, high net profit and build-up of native soil $\mathrm{P}$ in available form.

Ionic concentration in plant tissues, PUE and soil physical status. The higher concentrations of $\mathrm{P}, \mathrm{K}^{+}$and $\mathrm{Ca}^{2+}$ in $\mathrm{CR}$ treated plots could be due to enhanced mineral nutrition in soil through complete decomposition and mineralization of incorporated $\mathrm{CR}$ and $\mathrm{P}$ application further facilitated their intake by crop (Mahmood et al., 2013; Ali et al., 2012; Danga and Wakindiki, 2009). In particular, $\mathrm{P}$ application with $\mathrm{CR}$ incorporation considerably decreased $\mathrm{Na}^{+}$contents and increased $\mathrm{K}^{+}$and $\mathrm{Ca}^{2+}$ concentrations in wheat tissues (Table 5). Ali et al. (2003) have reported that in rhizosphere with higher nutrients concentration, plants absorbed and translocated relatively more $\mathrm{K}^{+}$and less $\mathrm{Na}^{+}$than at lower concentrations. The data indicated that, $\mathrm{P}$ application still at lower rate $\left(40 \mathrm{~kg} \mathrm{P}_{2} \mathrm{O}_{5} /\right.$ ha) along with $\mathrm{CR}$ incorporation performed better or even statistically equal to higher dose of $\mathrm{P}$ without $\mathrm{CR}$ incorporation. These findings could also be supported by the results of Mahmood et al. (2009), Delgado et al. (2002), Haq et al. (2001) and Kinraide (1999; 1998) who reported that salinity interferes with the absorption and translocation of $\mathrm{K}^{+}$and $\mathrm{Ca}^{2+}$ by plants.

It has been reported earlier that enhanced $\mathrm{CO}_{2}$ partial pressure in the rhizosphere during the processes of CR decomposition and microbial respiration increased the availability of $\mathrm{P}$ and other essential nutrients (Mahmood et al., 2013; Ali et al., 2012; Qadir et al., 2003). Crop residues incorporation upon decaying also modified the environment by adding organic matter and nutrients, supporting by the data in Table 1. Improved soil physical conditions owing to $\mathrm{CR}$ incorporation also enhanced P uptake.

Further increase in $\mathrm{P}$ application $\left(120 \mathrm{~kg} \mathrm{P}_{2} \mathrm{O}_{5} / \mathrm{ha}\right) \mathrm{did}$ not show significant difference in PUE by both (DSR and wheat) crops. On the other hand, under no CR incorporation, maximum PUE was determined from the treatments where $\mathrm{P}$ was applied at higher rate (120 $\mathrm{kg} \mathrm{P}_{2} \mathrm{O}_{5} / \mathrm{ha}$ ) and was not as much as of $80 \mathrm{~kg} \mathrm{P}_{2} \mathrm{O}_{5} / \mathrm{ha}$ application with $\mathrm{CR}$ incorporation. This was most probably due to excessive uptake of saline ions in the absence of CR that reduced crop biomass ultimately resulting in less PUE. Many studies have investigated the effects of $\mathrm{pH}$ variation on $\mathrm{P}$ retention and availability, but reliable enhancement in soil $\mathrm{P}$ availability has not yet been obtained. However, minor changes may be achievable. For example: evidences from many detailed studies show that the more organic matter there is in soil, the more the amount of very readily available plant P (Rehim et al., 2012; Leytem and Mikkelsen, 2005; Delgado et al., 2002; Manske et al., 2000).

\section{Conclusion}

Results of the present study of two years (2011 and 2012) indicated that $\mathrm{P}$ application @ 80 kg $\mathrm{P}_{2} \mathrm{O}_{5} /$ ha alongwith CR incorporation ( $2 \mathrm{t} / \mathrm{ha}$ ) was found to be superior than rest of the treatments in terms of producing maximum grain yields of both DSR and wheat crops grown under saline soil. During second year of DSR grown under $\mathrm{CR}$ incorporation, the lower $\mathrm{P}$ application rate (40 $\mathrm{kg} \mathrm{P}_{2} \mathrm{O}_{5} / \mathrm{ha}$ ) performed slightly better and produced comparable paddy yield as that of higher $\mathrm{P}$ application rate $\left(120 \mathrm{~kg} \mathrm{P}_{2} \mathrm{O}_{5} / \mathrm{ha}\right)$ without CR incorporation. Therefore, continuous $\mathrm{CR}$ incorporation is worth recommended to restore soil fertility as well as productivity. 


\section{References}

Abid, M., Ahmad, F., Ahmad, N., Ahmad, I. 2002. Effect of phosphorus on growth, yield and mineral composition of wheat in different textured saline sodic soils. Asian Journal of Plant Sciences, 1: 472.

Ahmad, N. 2002. Soil Fertility Management. Key to Food Security and Poverty Alleviation. In: Proceeding of $12^{\text {th }}$ National Congress of Soil Science Society, pp. 17-18, NIAB, Faisalabad, Pakistan.

Ahmad, N., Qureshi, R.H., Qadir, M. 1990. Irrigation Systems Management/Research (Biotic and Chemical Reclamation of Saline Sodic Soils). $4^{\text {th }}$ Annual Report, 1989-90. Department of Crop Physiology, University of Agriculture, Faisalabad, Pakistan.

Ali, A., Arshadullah, M., Hyder, S.I., Mahmood, I.A., Zaman, B. 2012. Rice productivity and soil health as affected by wheat residue incorporation along with nitrogen starter dose under salt-affected soil. Pakistan Journal of Agricultural Research, 25: 257-265.

Ali, A., Salim, M., Ahmad, I., Mahmood, I.A., Zaman, B., Sultana, A. 2003. Nutritional role of calcium on the growth of Brassica napus under saline conditions. Pakistan Journal of Agricultural Sciences, 40: 106-113.

Arshadullah, M., Ali, A., Hyder, S.I., Khan, A.M. 2012. Effect of wheat residue incorporation along with $\mathrm{N}$ starter dose on rice yield and soil health under saline sodic soil. The Journal of Animal \& Plant Sciences, 22: 753-757.

Aslam, M., Flowers, T.J., Qureshi, R.H., Yeo. A.R. 2008. Interaction of phosphate and salinity on the growth and yield of rice (Oryza sativa L.). Journal of Agronomy and Crop Science, 176: 249-258.

Byous, E.W., Williuams, J.E., Jonesa, G.E., Horwath, W.R., Kessel, C. 2004. Nutrient requirements of rice with alternative straw management. Better Crops, 36: 6-11.

Danga, B.O., Wakindiki, I.I.C. 2009. Effect of placement of straw mulch on soil conservation, nutrient accumulation, and wheat yield in a humid Kenyan highland. Journal of Tropical Agriculture, 47: 3036.

Delgado, A., Uceda, I., Andreu, L., Kassem, S. 2002. Fertilizer phosphorus recovery from gypsumamended reclaimed calcareous marsh soils. Arid Land Research Management, 16: 319-334.
Eagle, A.J., Bird, J.A., Horwath, W.R., Linquist, B.A., Brouder, S.M., Hill, J.E., Kessel, C.V. 2000. Rice yield and nitrogen utilization efficiency under alternative straw management practices. Agronomy Journal, 92: 1096-1103.

Fageria, N.K., Baligar, V.C., Jones, C.A. 1997. Growth and Mineral Nutrition of Field Crops, 656 pp., $2^{\text {nd }}$ edition, Marcel Dekker, Inc. NY, USA.

Ghafoor, A., Qadir, M., Murtaza, G. 2004. Salt-affected Soils: Principles of Management, 304 pp., Allied Book Centre, Urdu Bazar, Lahore, Pakistan.

Ghafoor, A., Shahid, M.I., Saghir, M., Murtaza, G. 1992. Use of high Mg brackish water on phosphogypsum and FYM treated saline-sodic soil. I. Soil improvement. Pakistan Journal of Agricultural Sciences, 29: 180-184.

Gomez, K.A., Gomez, A.A. 1984. Statistical Procedure for Agricultural Research, $2^{\text {nd }}$ edition, John Wiley and Sons, New York, USA.

GOP, 2014. Economic Survey of Pakistan 2013-14. Finance Division Government of Pakistan, Islamabad, Pakistan.

Haq, I., Khattak, S.G., Rehman, H., Ali, A., Salim, M. 2001. Effect of various amendments on the yield of rice crop under saline-sodic conditions in Mardan/Swabi District. International Journal of Agriculture Biology, 3: 289-291.

Kharub, A.S., Sharma, R.K., Mongia, A.D., Chhokar, R.S., Tripathi, S.C., Sharma, V.K. 2004. Effect of rice (Oryza sativa) straw removal, burning and incorporation on soil properties and crop productivity under rice-wheat (Triticum aestivum) system. Indian Journal of Agricultural Science, 74: 295299.

Kinraide, T.B. 1999. Interactions among $\mathrm{Ca}^{+2}, \mathrm{Na}^{+}$and $\mathrm{K}^{+}$in salinity toxicity: Quantitative resolution of multiple toxic and ameliorative effects. Journal of Experimental Botany, 50: 1495-1505.

Kinraide, T.B. 1998. Three mechanisms for the calcium alleviations of mineral toxicities. Plant Physiology, 118: $513-520$.

Krishna, G.M.A., Misra, A.K.K., Hati, K.M., Bandyopadhyay, K.K., Ghosh, P.K., Mohanty, M. 2004. Rice residue management options and effects on soil properties and crop productivity. Journal of Food, Agriculture and Environment, 2: 224-231.

Leytem, A.B., Mikkelsen, R.L. 2005. The nature of phosphorus in calcareous soils. Better Crops, 89: 11-13. 
Mahmood, I.A., Ali, A., Aslam, M., Shahzad, A., Sultan, T., Hussain, F. 2013. Phosphorus availability in different salt-affected soils as influenced by crop residue incorporation. International Journal of Agriculture and Biology, 15: 472-478.

Mahmood, I.A., Salim, M., Ali, A., Arshadullah, M., Zaman, B., Mir, A. 2009. Impact of calcium sulphate and calcium carbide on nitrogen use efficiency of wheat in normal and saline sodic soils. Soil \& Environment, 28: 29-37.

Mahmood, I.A., Aslam, M., Qureshi, R.H., Nawaz, S. 1994. Phosphorus nutrition of rice in a salt-affected soil fertilized with various nitrogen sources. Pakistan Journal of Agricultural Sciences, 31: 168173.

Manske, G.G.B., Ortiz-Monasterio, J.I., Van-Ginkel, M., Gonzalez, R.M., Fischer, R.A., Rajaram, S., Vlek, P.L.G. 2000. Importance of P uptake efficiency versus $\mathrm{P}$ utilization for wheat yield in acid and calcareous soils in Mexico. European Journal of Agronomy, 14: 261-274.

Maser, P., Gierth, M., Schroeder, J.I. 2002. Molecular mechanisms of potassium and sodium uptake in plants. Plant and Soil, 247: 43-54.

Minhas, P.S. 1996. Saline water management for irrigation in India. Review of Agricultural Water Management, 28: 273-288.

Mishra, B.N., Kumar, D., Shivay, Y.S. 2006. Effect of organic sources on productivity, grain quality and soil health of rice (Oryza sativa L.) - wheat (Triticum aestivum L.) cropping system. In: Extended Summaries of Golden Jubilee National Symposium on Conservation Agriculture and Environment, Ahlawat et al. (eds.), 280 pp., October 26-28, 2006, Banaras Hindu University, Varanasi, Organized by Indian Society of Agronomy and Banaras Hindu University, India.

Misra, R.D., Gupta, V.K., Pandey, D.S. 1996. Managing crop residue in rice. International Rice Research Notes, 21: 71-72.

NFDC, 2012. Fertilizer Use Related Statistics. National Fertilizer Development Centre, Planning Division, Government of Pakistan, Islamabad, Pakistan.

NFDC, 2010. Fertilizer Use Related Statistics. National Fertilizer Development Centre, Planning Division, Government of Pakistan, Islamabad, Pakistan.

Qadir, M., Steffens, D., Yan, F., Schubert, E. 2003. Sodium removal from a calcareous saline-sodic soil through leaching and plant uptake during phytoremediation. Land Degradation and Development, 14: 301-307.

Qayyum, M.A., Malik, M.D. 1988. Farm Production in Salt-Affected Soils. In: Managing Soil Resources. $I^{\text {st }}$ National Congress of Soil Science, pp. 356-364, Lahore, October 6-8, 1985. Lahore, Pakistan.

Qureshi, R.H., Qadir, M. 1992. Is the reclamation of dense saline sodic soils uneconomical? Myths and Facts. Pakistan Journal of Agricultural Sciences, 29: 317-318.

Rahmatullah, Gill, M.A., Shaikh, B.Z., Salim, M. 1994. Bioavailability and distribution of $\mathrm{P}$ among inorganic fractions in calcareous soils. Arid Soil Research and Rehabilitation, 8: 227-234.

Rath, A.K., Ramakrishnan, B., Rao, V.R., Sethunathan, N. 2005. Effects of rice-straw and phosphorus application on production and emission of methane from tropical rice soil. Journal of Plant Nutrition and Soil Science, 168: 248-254.

Rehim, A., Hussain, M., Abid, M., Zia-ul-Haq, M., Ahmad, S. 2012. Phosphorus use efficiency of Trititicum aestivum L. as affected by band placement of phosphorus and farmyard manure on calcareous soils. Pakistan Journal of Botany, 44: 1391-1398.

Rehim, A., Ranjha, A.M., Rahamtullah, Waraich, E.A. 2010. Effect of phosphorus application and irrigation scheduling on wheat yield and phosphorus use efficiency. Soil and Environment, 29: 15-22.

Rhoades, J.D. 1982. Cation Exchange Capacity. In: Methods of Soil Analysis. Part 2. Chemical and Microbiological Properties, A.L. Page (ed.), pp. 149158, American Society of Agronomy, Madison, Wisconsin, USA.

Ryan, J., Estefan, G., Rashid, A. 2001. Soil and Plant Analysis Laboratory Manual, pp. 172, International Center for Agricultural Research in the Dry Areas (ICARDA), Aleppo, Syria.

Sainio, P.P., Kontturi, M., Peltonen, J. 2006. Phosphorus seed coating enhancement on early growth and yield components in Oat. Agronomy Journal, 98: 206-211.

Saleem, M., Ahmad, T.N., David, J.G. 1986. Fertilizers and their Use in Pakistan. National Fertilizer Development Center, Planning and Development Division, Government of Pakistan, Islamabad, Pakistan. 
Sharma, S.N., Prasad, R. 2003. Yield and P uptake by rice and wheat grain in a sequence as influenced by phosphate fertilization with diammonium phosphate and Mussoorie rock phosphate with or without crop residue and phosphate solubilizing bacteria. Journal of Agricultural Sciences, 141: 359-369.

Shiva, D., Vyas, A.K., Shivay, Y.S., Kumar, D., Kumar, A. 2012. Yield of wheat and residual soil fertility under organic and inorganic fertilization. In: Proceedings of National Seminar on Indian Agriculture: Preparedness for Climate Change, S. Dhar, S. Barthakur, K. Prajapati, K. Annapurna, T. Satyavathi and A. K. Vyas (ed.), pp. 139-140, March 24-25, 2012, New Delhi, Organized by Indian Society of Agricultural Science, Division of Agro- nomy, IARI, New Delhi, India.

Slaton, N.A., Wilson Jr., C.E., Norman, R.J., Ntamatungiro S., Frizzell, D.L. 2002. Rice response to phosphorus fertilizer application rate and timing on alkaline soils in Arkansas. Agronomy Journal, 94: 1393-1399.

Van der Eijk, D., Janssen, B.H., Oenema, O. 2006. Initial and residual effects of fertilizer phosphorus on soil phosphorus and maize yields on phosphorus fixing soils. A case study in Southwest Kenya. Agricultural Ecology Management, 116: 104-12. Yadvinder, S., Bijay, S., Ladha, J.K., Khind, C.S., Khera, T.S., Bueno, C.S. 2004. Effects of residue decomposition on productivity and soil fertility in rice-wheat rotation. Soil Science Society of American Journal, 68: $854-864$. 